\title{
CONVOLUTION POWERS OF SINGULAR-SYMMETRIC MEASURES. II
}

\author{
KEIJI IZUCHI
}

\begin{abstract}
Let $G$ be an infinite compact abelian group such that its dual group contains an infinite independent subset. $\mathcal{L}(G)$ denotes the sum of all radicals of group algebras contained in the measure algebra on $G$. Then, for a positive integer $k$, there is a measure $\mu$ on $G$ such that $\mu^{n}$ is singularsymmetric for $1<n<k$ and $\mu^{n} \in \mathcal{L}(G)$ for $n>k$.
\end{abstract}

Let $G$ be an infinite compact abelian group and $\hat{G}$ its dual group. A subset $E$ of $G$ is said to be independent if for every choice of distinct points $x_{1}, \ldots, x_{i}$ of $E$ and integers $n_{1}, \ldots, n_{i}$, either $n_{1} x_{1}=n_{2} x_{2}=\cdots=n_{i} x_{i}=0$ or $n_{1} x_{1}+n_{2} x_{2}+\cdots+n_{i} x_{i} \neq 0$. In this paper, we assume that $\hat{G}$ contains an infinite independent set. Let $M(G)$ be the measure algebra on $G$ and $L^{1}(G)$ the group algebra on $G$. We denote by $\operatorname{Rad} L^{1}(G)$ the radical of $L^{1}(G)$ in $M(G)$. We put $\mathcal{L}(G)=\Sigma_{\tau} \operatorname{Rad} L^{1}\left(G_{\tau}\right)$, where $\tau$ runs through over L.C.A. group topologies on $G$ which are stronger than the original one. We denote by $\Delta$ the maximal ideal space of $M(G)$. For $\mu \in M(G)$, we put $\mu^{*}(E)=\overline{\mu(-E)}$ for every Borel subset $E$ of $G$. We denote by $\mathfrak{M}$ the set of all symmetric measures of $M(G)$, that is, $\mathfrak{M}=\left\{\mu \in M(G) ; \tilde{\mu}^{*}(f)=\overline{\tilde{\mu}(f)}\right.$ for every $f \in$ $\Delta$ \}, where $\tilde{\mu}$ is the Gel'fand transform of $\mu$. Then we have $\mathcal{L}(G) \subset \mathfrak{M}$. A measure $\mu \in \mathfrak{M}$ is called singular-symmetric if $\mu$ is singular with $\mathcal{L}(G)$ $(\mu \perp \mathcal{L}(G))$.

In [1] and [2], the author showed that there exists a singular-symmetric measure on $\bar{R}$ such that $\mu * \mu \in \mathcal{L}(\bar{R})$, where $\bar{R}$ is the Bohr compactification of the real line $R$. In [5], Shimizu showed that there exists a singular-symmetric measure on $H=\Pi H_{n}$, where $H_{n}$ is an infinite compact abelian group $(n=1,2, \ldots)$, using essentially the same method as in [1]. In the previous paper [3], the author showed that there exists a singular-symmetric measure $\mu$ on $\bar{R}$ such that $\mu^{n}$ is singular-symmetric for every positive integer $n$, where $\mu^{n}=\mu^{n-1} * \mu$ and $\mu^{1}=\mu(n \geqslant 2)$.

In this paper, we show the following. The essential idea of the proof is found in [1].

THEOREM. Let $G$ be a compact abelian group whose dual group $\hat{G}$ contains an infinite independent set. Then, for each positive integer $k$, there exists a

Received by the editors February 2, 1976.

AMS (MOS) subject classifications (1970). Primary 43A05, 43A10, 43A32.

Key words and phrases. Convolution powers, singular-symmetric, measure algebra.

- American Mathematical Society 1977 
singular-symmetric measure $\mu$ on $G$ such that

(a) $\mu^{n}$ is singular-symmetric if $1 \leqslant n \leqslant k$,

(b) $\mu^{n} \in \mathcal{L}(G)$ if $n>k$.

The proof of the Theorem proceeds in steps.

(I) We fix a positive integer $k$. Let $E$ be an infinite countable independent subset of $\hat{G}$.

Lemma. There exists a family of infinite subsets $\left\{E_{n, i}\right\}_{0<n<\infty ; 1<i<2^{n}}$ of $E$ such that:

(1) $E_{n, i}$ is an infinite subset of $E$ for $0 \leqslant n<\infty$ and $1 \leqslant i \leqslant 2^{n}$;

(2) $E_{n, i} \varsubsetneqq E_{n+1,2 i-1}$ and $E_{n, i} \subsetneq E_{n+1,2 i}$ for $0 \leqslant n<\infty$ and $1 \leqslant i \leqslant 2^{n}$;

(3) $E_{n} \cap E_{n+1,2 i-1}=E_{n} \cap E_{n+1,2 i}=E_{n, i}$, where $E_{n}=\bigcup\left\{E_{n, i} ; 1 \leqslant i \leqslant\right.$ $\left.2^{n}\right\}$, for $0 \leqslant n<\infty$ and $1 \leqslant i \leqslant 2^{n}$;

(4) for $s>n>k$ and $1 \leqslant i_{1}<i_{2}<\cdots<i_{n} \leqslant 2^{s}$, we have

$$
E_{s-1} \cap\left(\bigcap_{q=1}^{n} E_{s, i_{q}}\right)=\bigcap_{q=1}^{n} E_{s, i_{q}} ;
$$

(5) for $s>k \geqslant n_{1} \geqslant n_{2}, 1 \leqslant i_{1}<i_{2}<\cdots<i_{n_{1}} \leqslant 2^{s}$ and $1 \leqslant j_{1}<j_{2}$ $<\cdots<j_{n_{2}} \leqslant 2^{s}$, we have

$$
\left(\bigcap_{q=1}^{n_{2}} E_{s, j_{q}} \backslash E_{s-1}\right) \backslash\left(\bigcap_{q=1}^{n_{1}} E_{s, i_{q}} \backslash E_{s-1}\right)
$$

is an infinite set if $\left\{j_{1}, j_{2}, \ldots, j_{n_{2}}\right\} \underset{f}{\subsetneq}\left\{i_{1}, i_{2}, \ldots, i_{n_{1}}\right\}$,

$$
\left(\bigcap_{q=1}^{n_{2}} E_{s, j_{q}} \backslash E_{s-1}\right) \backslash\left(\bigcap_{q=1}^{n_{1}} E_{s, i_{q}} \backslash E_{s-1}\right)
$$

and

$$
\left(\bigcap_{q=1}^{n_{1}} E_{s, i_{q}} \backslash E_{s-1}\right) \backslash\left(\bigcap_{q=1}^{n_{2}} E_{s, j_{q}} \backslash E_{s-1}\right)
$$

are infinite sets if $\left\{j_{1}, j_{2}, \ldots, j_{n_{2}}\right\} \not \subset\left\{i_{1}, i_{2}, \ldots, i_{n_{1}}\right\}$.

Proof. This Lemma is an elementary set-theoretic fact about infinite sets, so we do not give the detailed proof. In the next step, we construct a measure $\mu$ on $G$ using the properties of $\left\{E_{n, i}\right\}_{n, i}$ of the Lemma, and in Steps (III) and (IV) we show that $\mu$ satisfies conditions (a) and (b) of the Theorem. Property (4) is used to show (a) and property (5) is used to show (b). In [1], we constructed a measure $\mu$ on $G$ such that $\mu \perp \mathcal{L}(G)$ and $\mu * \mu \in \mathcal{L}(G)$, using a certain family of subsets $\left\{E_{\alpha}\right\}_{\alpha \in \Lambda}$ of $E$. Lemma 1 of [1] is the case $k=1$ of this Lemma. In [3], we constructed a measure $\mu$ on $G$ such that $\mu^{n}$ is singular-symmetric for every positive integer $n$, using a certain family of subsets $\left\{E_{n, i}\right\}_{n, i}$ of $E$. But the properties of $\left\{E_{n, i}\right\}$ in [3] differ extremely from those in this Lemma. 
(II) Let $H_{n, i}$ be the subgroup of $\hat{G}$ generated by $E_{n, i}$, and $H_{n}=\cup\left\{H_{n, i}\right.$; $\left.1 \leqslant i \leqslant 2^{n}\right\}$. Then we have:

(6) for $0 \leqslant n<\infty$ and $1 \leqslant i \leqslant 2^{n}, H_{n} \cap H_{n+1,2 i-1}=H_{n} \cap H_{n+1,2 i}=$ $H_{n, i}$ by (3);

(7) for $s \geqslant n>k$ and $1 \leqslant i_{1}<i_{2}<\cdots<i_{n} \leqslant 2^{s}$, we have

$$
\begin{gathered}
\bigcap_{q=1}^{n}\left(\bigcup_{t>s}\left(\bigcup\left\{H_{t, j} ; 2^{t-s}\left(i_{q}-1\right)+1 \leqslant j \leqslant 2^{t-s_{i}}\right\}\right)\right) \\
=\bigcap_{q=1}^{n} H_{s, i_{q}} \text { by (4). }
\end{gathered}
$$

Let $G_{n, i}=H_{n, i}^{\perp}$ be the annihilator of $H_{n, i}$ in $G$. Then $\left\{G_{n, i}\right\}_{n, i}$ are compact subgroups of $G$ and $\left(\cap_{t=1}^{q} H_{n, i_{i}}\right)^{\perp}=\bigoplus_{1<t<q} G_{n, i_{i}}$, where

$$
\bigoplus_{1<t<q} G_{n, i_{i}}=\left\{x_{1}+x_{2}+\cdots+x_{q} ; x_{t} \in G_{n, i_{i}}(t=1,2, \ldots, q)\right\} \text {. }
$$

We put

$$
\mu_{n}=\sum_{i=1}^{2^{n}}\left(\frac{1}{2}\right)^{n} m\left(G_{n, i}\right),
$$

where $m\left(G_{n, i}\right)$ is the normalized Haar measure on $G_{n, i}$; then $\mu_{n} \geqslant 0$ and $\left\|\mu_{n}\right\|=1$. For $\gamma \in H_{s} \backslash H_{s-1}$, we put $\beta_{\gamma}=\operatorname{card}\left\{i ; 1 \leqslant i \leqslant 2^{s}, \gamma \in H_{s, i}\right\}$. Then we have $\hat{\mu}_{s}(\gamma)=\beta_{\gamma}\left(\frac{1}{2}\right)^{s}$ by (8), where $\hat{\mu}_{s}$ is the Fourier-Stieltjes transform of $\mu_{s}$. By (6), we have $\operatorname{card}\left\{i ; 1 \leqslant i \leqslant 2^{n}, \gamma \in H_{n, i}\right\}=2^{n-s} \beta_{\gamma}$ for $n>s$. Then $\hat{\mu}_{n}(\gamma)=\left(\frac{1}{2}\right)^{n} 2^{n-s} \beta_{\gamma}=\left(\frac{1}{2}\right)^{s} \beta_{\gamma}=\hat{\mu}_{s}(\gamma)$ for every $n>s$. If $\gamma \notin \cup_{s=0}^{\infty} H_{s}$, then $\hat{\mu}_{n}(\gamma)=0$ for $n=0,1,2, \ldots$. These imply that $\left\{\mu_{n}\right\}_{n=0}^{\infty}$ has only one weak*-cluster point $\mu$ in $M(G)$, and $\mu \geqslant 0,\|\mu\|=1$.

(III) We show that $\mu$ satisfies condition (a) of our Theorem. We fix a positive integer $n$ such that $n>k$ and prove (a). For $s \geqslant k$ and $1 \leqslant i \leqslant 2^{s}$, we put $\lambda_{s, i, t}=\left(\frac{1}{2}\right)^{t} \sum\left\{m\left(G_{t, j}\right) ; 2^{t-s}(i-1)+1 \leqslant j \leqslant 2^{t-s} i\right\}$ for $t \geqslant s$. Then $\left\{\lambda_{s, i, t}\right\}_{t=s}^{\infty}$ has only one weak*-cluster point $\lambda_{s, i}$ in $M(G)$ in the same way as the previous part, and we have:

(9) $\mu=\Sigma_{i=1}^{2^{s}} \lambda_{s, i}$ for $s=1,2, \ldots$;

(10) $\lambda_{s, i}$ is concentrated on $G_{s, i}$ for $s \geqslant k$ and $1 \leqslant i \leqslant 2^{s}$;

(11) $\hat{\lambda}_{s, i}(\gamma)=\left(\frac{1}{2}\right)^{s}$ for $\gamma \in H_{s, i}$;

(12) $\hat{\lambda}_{s, i}(\gamma) \neq 0$ for $\gamma \in \cup_{t>s}\left(\cup\left\{H_{t, j} ; 2^{t-s}(i-1)+1 \leqslant j \leqslant\right.\right.$ $\left.\left.2^{t-s_{i}}\right\}\right), \hat{\lambda}_{s, i}(\gamma)=0$ for other $\gamma$ in $\hat{G}$.

For $s \geqslant n>k$ and $1 \leqslant i_{1}<i_{2}<\cdots<i_{n} \leqslant 2^{s}$, we have $\hat{\lambda}_{s, i_{q}}(\gamma)=0$ for $\gamma \notin \cap_{q=1}^{n}\left(\cup_{t \geqslant s}\left(\cup\left\{H_{t, j} ; 2^{t-s}\left(i_{q}-1\right)+1 \leqslant j \leqslant 2^{t-s_{i}}\right\}\right)\right)$ by (12). By (7), we have $\hat{\lambda}_{s, i_{q}}(\gamma)=0$ for $\gamma \notin \cap_{q=1}^{n} H_{s, i_{q}}$. By (11), we have $\Pi_{q=1}^{n} \hat{\lambda}_{s, i_{q}}=\left(\frac{1}{2}\right)^{n s}$ on $\cap_{q=1}^{n} H_{s, i_{q}}$, so that

$$
\stackrel{*}{*} \lambda_{s=1}=\left(\frac{1}{2}\right)^{n s} m\left(\underset{1<q<n}{\bigoplus_{q}} G_{s, i_{q}}\right) \in \mathcal{L}(G) .
$$

We put $\mu^{n}=\left(\sum_{i=1}^{2^{s}} \lambda_{s, i}\right)^{n}=P_{s}+P_{s}^{\prime}$, where $P_{s}=\sum\left\{n ! *_{q=1}^{n} \lambda_{s, i_{q}} ; \quad 1 \leqslant i_{1}\right.$ 
$\left.<\cdots<i_{n} \leqslant 2^{s}\right\}$ and $P_{s}^{\prime}$ includes squares and higher powers of the $\lambda_{s, i}$. Here we note the following fact:

$$
\begin{aligned}
& \left\|\sum\left\{n ! \underset{q=1}{*} \lambda_{s, i_{q}} ; 1 \leqslant i_{1}<i_{2}<\cdots<i_{n} \leqslant 2^{s}\right\}\right\|=\left(\begin{array}{l}
2^{s} \\
n
\end{array}\right) n !\left(\frac{1}{2}\right)^{n s} \\
& \rightarrow 1 \quad(s \rightarrow \infty) \text { for every positive integer } n \text {. }
\end{aligned}
$$

Since $1=\left\|\mu^{n}\right\|=\left\|P_{s}\right\|+\left\|P_{s}^{\prime}\right\|$ and $\left\|P_{s}\right\| \rightarrow 1(s \rightarrow \infty)$ by (14), we have $\left\|P_{s}^{\prime}\right\|$ $\rightarrow 0$. Therefore $P_{s} \rightarrow \mu^{n}(s \rightarrow \infty)$ in norm. Since $\mathcal{L}(G)$ is norm closed and $P_{s} \in \mathcal{L}(G)(s \geqslant n>k)$ by (13), we have $\mu^{n} \in \mathcal{L}(G)(n>k)$. This completes the proof of (a).

(IV) We fix a positive integer $n$ such that $1 \leqslant n \leqslant k$, and prove (b). Suppose that $\mu^{n}, \mathcal{L}(G)$. Then there is a L.C.A. group topology $\tau$ on $G$ which is stronger than the original one such that $\mu^{n} \not \operatorname{Rad} L^{1}\left(G_{\tau}\right)$. Also, this implies $\mu^{k+1} \not \operatorname{Rad} L^{1}\left(G_{\tau}\right)$. We put $\mu^{k+1}=\eta_{1}+\eta_{2}$ and $\varepsilon=\left\|\eta_{1}\right\|>0$, where $\eta_{1}$ is the part of $\mu^{k+1}$ which is contained in $\operatorname{Rad} L^{1}\left(G_{\tau}\right)$ and $\eta_{1} \perp \eta_{2}$. By (14), there is a sufficient large positive integer $s$ such that $s>k$ and

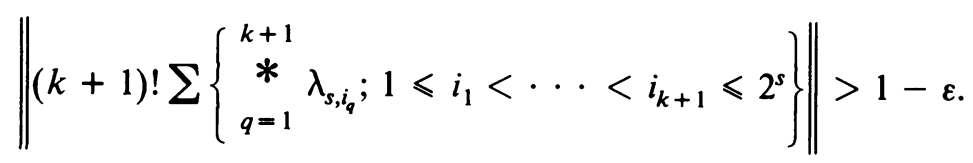

Then there exists $\left(j_{1}, j_{2}, \ldots, j_{k+1}\right)$ such that $1 \leqslant j_{1}<\cdots<\lambda j_{k+1} \leqslant 2^{s}$ and $*_{q=1}^{k+1} \lambda_{s j_{q}}, \chi \operatorname{Rad} L^{1}\left(G_{\tau}\right)$. Then by (13), $\tau$ is the topology on $G$ that makes $\bigoplus_{1<q<k+1}^{q-1} G_{s, j_{q}}$ an open compact subgroup. We put

$$
\begin{aligned}
& \mathscr{1}=\{<k+1 \\
& =\left\{\left(i_{1}, i_{2}, \ldots, i_{n}\right) ; 1 \leqslant i_{1} \leqslant i_{2} \leqslant, \ldots, \leqslant i_{n} \leqslant 2^{s}\right\}, \\
& Q_{1}=\left\{\left(i_{1}, \ldots, i_{n}\right) \in Q ;\left\{i_{1}, \ldots, i_{n}\right\} \subset\left\{j_{1}, j_{2}, \ldots, j_{k+1}\right\}\right\}, \\
& Q_{2}=\left\{\left(i_{1}, \ldots, i_{n}\right) \in Q ;\left\{i_{1}, \ldots, i_{n}\right\} \not \subset\left\{j_{1}, j_{2}, \ldots, j_{k+1}\right\}\right\} .
\end{aligned}
$$

If $\left(i_{1}, \ldots, i_{n}\right) \in Q_{1}$, then $\cap_{q=1}^{k+1} H_{s, j_{q}} \subsetneq \cap_{q=1}^{n} H_{s, i_{q}}$, and this implies that

$$
\underset{1<q<k+1}{\bigoplus_{s, j_{q}}}=\left(\bigcap_{q=1}^{k+1} H_{s, j_{q}}\right)^{\perp} \supsetneqq\left(\bigcap_{q=1}^{n} H_{s, i_{q}}\right)^{\perp}=\bigoplus_{1<q<n} G_{s, i_{q}},
$$

and $\bigoplus_{1<q<k+1} G_{s, j_{q}} / \bigoplus_{1 \leqslant q<n} G_{s, i_{q}}$ is an infinite compact group by (4) and (5). Then we have $m_{\tau}\left(\bigoplus_{1<q \leqslant n} G_{s, i, q}\right)=0$, where $m_{\tau}$ is a Haar measure on $G_{\tau}$. If $\left(i_{1}, \ldots, i_{n}\right) \in Q_{2}$, then $\cap_{q=1}^{k+1} H_{s, j_{q}} \supset \cap_{q=1}^{n} H_{s, i_{q}}$, and this implies also $m_{\tau}\left(\oplus_{1<q<n} G_{s, i_{q}}\right)=0$ by (4) and (5), so that we have $m_{\tau}\left(\oplus_{1<q \leqslant n} G_{s, i_{q}}\right)=0$ for $\left(i_{1}, \ldots, i_{n}\right) \in Q$. Since

$$
\mu^{n}=\left(\sum_{i=1}^{2^{s}} \lambda_{s, i}\right)^{n}=\sum\left\{b\left(i_{1} \cdots i_{n}\right) \underset{q=1}{*} \lambda_{s, i_{q}} ;\left(i_{1}, \ldots, i_{n}\right) \in Q\right\},
$$

where $b\left(i_{1}, \ldots, i_{n}\right)$ is a positive integer, the support of $\mu^{n}$ is contained in 
$\cup\left\{\bigoplus_{1<q<n} G_{s, i_{q}} ;\left(i_{1}, \ldots, i_{n}\right) \in Q\right\}$ by (10). By the above facts, we have $m_{\tau}\left(\cup\left\{\oplus_{1<q<n} G_{s, i_{q}} ;\left(i_{1}, \ldots, i_{n}\right) \in Q\right\}\right)=0$. This implies that $\mu^{n} \perp L^{1}\left(G_{\tau}\right)$. Let $\left(i_{1}, \ldots, i_{n}\right) \in Q$ and $p>1$. Then $(10)$ implies that $\left(*_{q-1}^{n} \lambda_{s, i_{q}}\right)^{p}$ is supported in $\bigoplus_{1<q<n} G_{s, i_{q}}$. Therefore $\left(*_{q=1}^{n} \lambda_{s, i_{q}}\right)^{p} \perp L^{1}\left(G_{\tau}\right)$. This shows that $\left(*_{q=1}^{n} \lambda_{s, i_{q}}\right) \perp \operatorname{Rad} L^{1}\left(G_{\tau}\right)[6]$. Then we have $\mu^{n} \perp \operatorname{Rad} L^{1}\left(G_{\tau}\right)$. This is a contradiction. This completes the proof of (b).

Remark 1. For $\lambda \in M(G)$, we denote by $\sigma(\lambda)$ the spectrum of $\lambda$, that is, $\sigma(\lambda)=\{\tilde{\lambda}(f) ; f \in \Delta\}$. Let $\mu$ be the measure that is constructed in our Theorem. Then $\sigma(\mu)$ is a countable set and there are no nonzero elements in $\sigma(\mu)$ that are cluster points of $\sigma(\mu)$.

REMARK 2. For fixed positive integers $k<q$, there are $\mu_{1}, \mu_{2}, \ldots, \mu_{q} \in$ $M(G)$ such that $\left(\mu_{i}\right)^{s}$ is singular-symmetric for $1 \leqslant s \leqslant k$ and $\left(\mu_{i}\right)^{s} \in \mathcal{L}(G)$ for $s>k(i=1,2, \ldots, q)$, and $*_{i=1}^{q} \mu_{i}$ is singular-symmetric. Because, for an infinite independent subset $E$ of $\hat{G}$, let $E^{\prime}$ be an infinite subset of $E$ such that $E \backslash E^{\prime}$ is an infinite subset. We construct $\mu_{1} \in M(G)$ such that $\left(\mu_{1}\right)^{s} \perp \mathcal{L}(G)$ for $1 \leqslant s \leqslant k,\left(\mu_{1}\right)^{s} \in \mathcal{L}(G)$ for $s>k$, and $\hat{\mu}_{1}=0$ on $\hat{G} \backslash H^{\prime}$, where $H^{\prime}$ is the subgroup of $\hat{G}$ generated by $E^{\prime}$. Also, we construct $\mu_{2}, \ldots, \mu_{q}$ $\in M(G)$ such that $\left\|\mu_{i}\right\|=1,\left(\mu_{i}\right)^{s} \perp \mathcal{L}(G)$ for $1 \leqslant s \leqslant k$ and $\left(\mu_{i}\right)^{s} \in \mathscr{L}(G)$ for $s>k$ and $\hat{\mu}_{i}=1$ on $H^{\prime}(i=2,3, \ldots, q)$. Then $*_{i=1}^{q} \mu_{i}=\mu_{1} \perp \mathcal{L}(G)$.

REMARK 3. For the circle group, there cannot exist any measure such as we have constructed in out Theorem. (This is an elementary argument.) Whether there exist any singular-symmetric measures on the circle (necessarily with all their powers singular) remains an open question.

The author would like to express his thanks to the referee for his kind advice.

\section{REFERENCES}

1. K. Izuchi, On a problem of J. L. Taylor, Proc. Amer. Math. Soc. 53 (1975), 347-352.

2 . $\_$A remark on a certain measure in $\operatorname{Rad} L^{1}(G)$, Rep. Fac. Engrg. Kanagawa Univ. 14 (1976), 1-2.

3. Convolution powers of singular-symmetric measures (to appear).

4. W. Rudin, Fourier analysis on groups, Interscience Tracts in Pure and Appl Math., no. 12, Interscience, New York, 1962. MR 27 \#2808.

5. T. Shimizu, Independent sets and measure algebras (to appear).

6. J. L. Taylor, Convolution measure algebras with group maximal ideal spaces, Trans. Amer. Math. Soc. 128 (1967), 257-263. MR 35 \#3375.

7. __ L-subalgebras of $M(G)$, Trans. Amer. Math. Soc. 135 (1969), 105-113. MR 38 \# 1472 .

8. , Measure algebras, CBMS Regional Conf. Ser. in Math., no. 16, Amer. Math. Soc., Providence, R.I., 1973.

Department of Mathematics, Kanagawa University, Yokohama, Japan 